ACTA THERIOLOGICA

Vol. 25, 10: 105-114, 1980

\title{
Der Steinmarder Martes foina (Erxleben, 1777) in der Mongolei ${ }^{t}$
}

\author{
Namshil CHOTOLCHU, Michael STUBBE \& Naniragijn DAWAA
}

Chotolchu N., Stubbe M. \& Dawaa N., 1980: Der Steinmarder Martes foina (E rx le be n, 1777) in der Mongolei. Acta theriol., 25, 10: 105-114 [Mit 4 Tabellen, 2 Abb. und Tafeln IV-V].

(The stone marten Martes foina ( $\mathrm{Erxleben,} \mathrm{1777)} \mathrm{in} \mathrm{Mongolia)}$

Stone martens are great rarities in mammal collections from Mongolia. Beside of biometric dates of 17 specimens, collected between 1974 and 1978, there are new information of distribution and ecology of Martes foina in MPR. The distribution of Martes foina is reaching from the southern parts of Mongolia to the northern zone with Januaryisotherms about $-25^{\circ} \mathrm{C}$. The species was regularly and commonly met in SW-Mongolia at Bulgan-gol, in the Mongolian Altai in the area of the Somons Zogt and Bugat, as well as $20 \mathrm{~km}$ northern of the town Chovd (Kobdo) in the Ulan-ul-mountains. Pikas, little rodents and birds are the mein food of martens. First reproduction data are noted.

[Zool. Inst. Mongol. Acad. Sci. Ulan-Bator, MPR (NC); Dept. of Zool., Section Biosciences, Martin-Luther-University Halle, DDR-402 Halle, Domplatz 4 (MS); Dept. of Zool. State University, Ulan-Bator, MPR (ND)].

Der Steinmarder gehört in Zentralasien $\mathrm{zu}$ den wenig bekannten und kaum untersuchten Raubsäugern. Taxonomisch werden die Belege aus der MVR bisher der Subspezies Martes foina intermedia (S e ve r t z o v, 1873) mit der Terra typica Karagodshur in Quellgebiet des Flusses Tschu südlich des Issyk-Kul zugeordnet. Die Unterart bewohnt die Gebirge Mittelasiens vom Kopet-Dag und Bolschoi Balchan bis zum südlichen Altai, wo es nach Strog a nov (1962) Nachweise aus den Tälern der Flüsse Ulba, Buchtarma und Kurtschum gibt. Als Siedlungsgebiete werden ferner für die asiatische Sowjetunion genannt: Karatau, Tienschan, Talasker und Sailisker Alatau, Kirgisengebirge, Westpamir, Dzungarischer Alatau, Saure und Tarbagatai. Das Areal schließt außerhalb der SU den Nordwestiran, Afghanistan, West-Pakistan, Westhimalaja, den Chinesischen Tienschan, Tibet und die an den Altai angrenzenden Gebiete der Mongolischen Volksrepublik ein (H e p tne r \& N a u m ov, 1974).

1 Ergebnisse der Mongolisch-Deutschen Biologischen Expeditionen seit 1962, Nr. 85 gewidmet unserem Kollegen und Mongoleiforscher Dr. Rudolf Piechocki, Kustos am Wissenschaftsbereich Zoologie der Sektion Biowissenschaften der MartinLuther-Universität Halle, mit den besten Wünschen zum 60. Geburtstag am 8.11.1979. 
Das Vorkomen in der Mongolei wird nach B a n nikov (1954) erstmals von Pevzov 1879 (und 1883) erwähnt, und zwar von der Südabdachung des Mongolischen Altai und als selten für den Changaj bezeichnet. B a n n i k ov (1954) berichtet weiter, daß P o t a n in (1881) den Steinmarder aus der Umgebung von Chovd (Kobdo) and Uljasutai nennt, wo jährlich bis zu 200 Bälge gehandelt wurden. Dagegen galt er als selten für das Darchatsker Gebiet im Norden, wo jährlich nur 2 bis 3 Felle anfielen. Ein Vorkommen im Großen Chingan, wie es wohl von

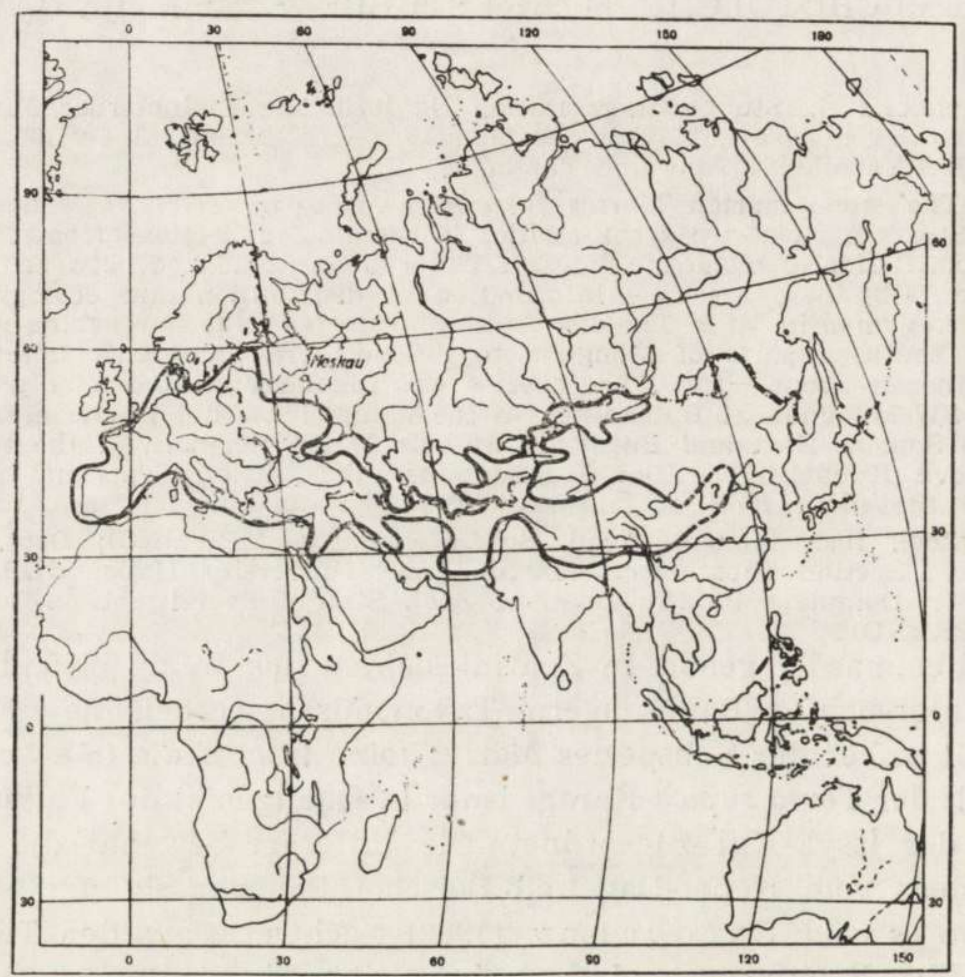

Abb. 1. Verbreitung des Steinmarders Martes foina in Eurasien (nach He pt ner \& $\mathrm{N}$ a u mov, 1974).

B o r o d o v ski (1894) vermutet wurde, wird zu Recht von B a n i k ov (1954) stark bezweifelt. Aus der Stadt Chovd brachte 1899 auch K o z l o v einen Balg mit nach Petersburg.

B a nnikov (1954), der Anfang der 40er Jahre in der MVR tätig war, nennt den Steinmarder für den Mongolischen Altai als große Seltenheit mit Einzelvorkommen in den westlichsten Gebirgszügen, die zum Russischen Altai Anschluß gewinnen. Ein Vorkommen im GobiAltai - im Ich-bogd-Massiv, Tost-ul, Solon-ul und Gurban-Sajchan - ist trotz Aussagen ansässiger Jäger, nicht bestätigt worden, aber auch nicht 
auszuschließen. Nach Chotolchu liegt vom Gurban-Sajchan ein Fundschädel vor, den eine der letzten Mongolisch-Sowjetischen Expeditionen mitbrachte. Für eine Besiedlung des Changaj fand B a nn ikov keine neuen Hinweise, wohl aber konnte er Beobachtungen für das Zagan-bogd-Gebirge in der Transaltaigobi verbuchen. Außerdem konnte an Hand von Bälgen in dem Ort Chatchal, das seltene Vorkommen in Chuvsgul-Gebiet erneut von ihm belegt weren.

Sowohl Dulamseren (1970) als auch Dulamseren \& Chot o l c h u (1972) erwähnen den Steinmarder für den Changaj ohne Belege

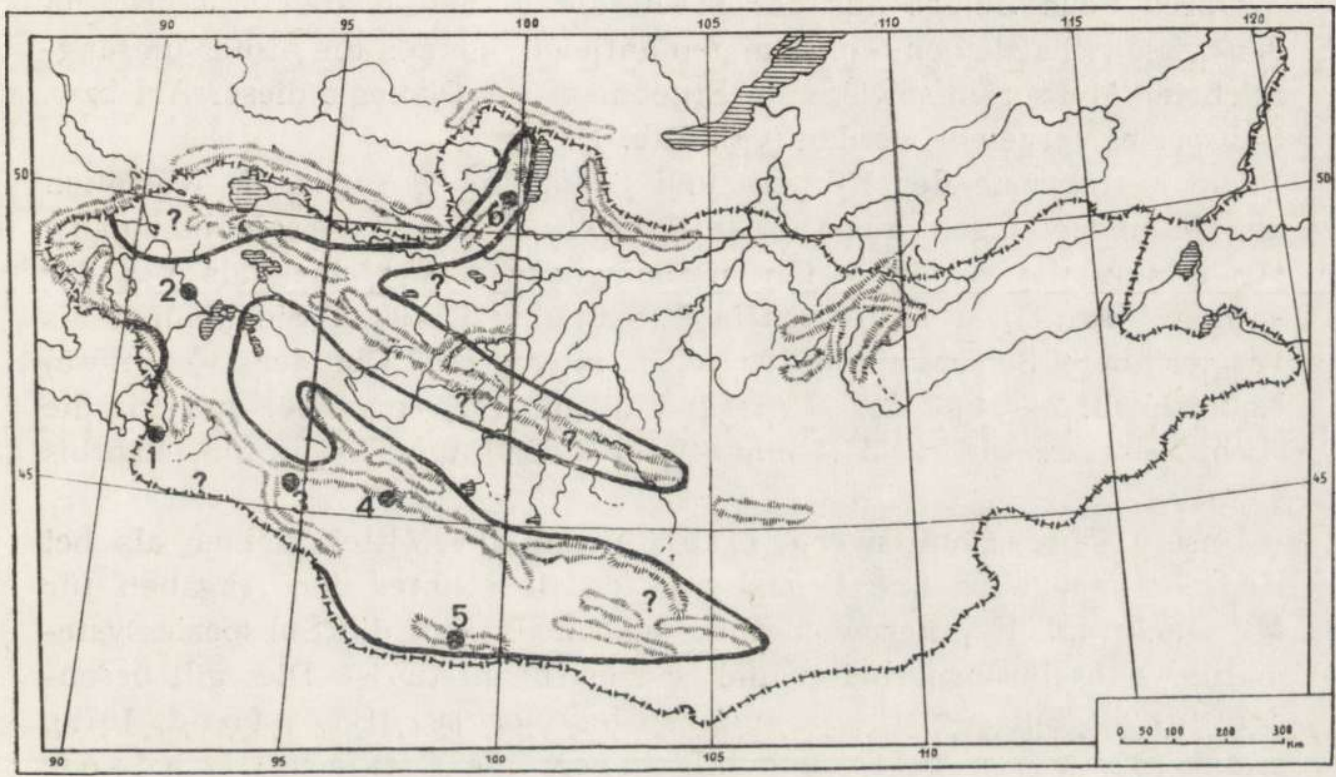

Abb. 2. Die Verbreitung von Martes foina in der Mongolischen Volksrepublik mit 1 - Bulgan-gol, 2-Chovd-gol, Ulan-ul, 3-Somon Bugat, 4-Somon Zogt, 5Zagan-bogd-ul, 6-Somon Chatchal.

anzuführen. Letztere weisen darauf hin, daß Martes foina in der Mongolei neuerdings häufiger geworden sei. Wie selten bisher Untersuchungsmaterial dieser Subspezies in wissenschaftliche Sammlungen gekommen ist, zeigt sich daran, daß B a n nik ov (1954) kein eigenes Belegmaterial zur Verfügung stand und daß $\mathrm{H}$ e ptne r \& $\mathrm{Na} \mathrm{u} \mathrm{mov}$ (1974) in ihrem kapitalen Werk nur die Schädel von 5 ô und $4 \%$ aus dem sowjetischen Areal auswerten konnten. B a n n i k ov konnte ermitteln, daß das Fellaufkommen dieser Art in der MVR in den $30 \mathrm{er}$ und $40 \mathrm{er}$ Jahren dieses Jahrhunderts die jährliche Anzahl von 20 bis 30 Bälgen 
nicht überschritt. Dies war sicher auch ein Grund dafür, daß der Steinmarder in der Jagdgesetzgebung der MVR unter strengen abscluten Schutz gestellt wurde.

R y a b ov (1976) konnte für den mittleren Teil der Sowjetunion in den letzten Jahrzehnten für den Steinmarder eine Arealerweiterung feststellen. Nach B a ke v (1972) ist das Areal des thermophilen Steinmarders etwa koinzident mit der $-10^{\circ} \mathrm{C}-$ Januarisotherme. Dies trifft nach den Ergebnissen der Biologischen Expeditionen der MVR/DDR im nordwestlichen Zentralasien nicht zu. Dort erreicht das Areal Januarisothermen von $-25^{\circ} \mathrm{C}$ und darunter.

Durch neue Belege aus der MVR, die in den letzten 5 Jahren in den wissenschaftlichen Sammlungen anfielen, können die bisher umfangreichsten Meßserien sowie erste Ergebnisse zur Ökologie dieser Art bzw. Subspezies vorgelegt werden (vgl. Tab. 1).

Die Mittelwerte der Körper- und Schädelmaße sowie die Variation der Geschlechter geht aus Tab. 2 hervor. Nach $\mathrm{H}$ e p tn e r \& N a u m ov (1974) sind die Gesamtmaße anscheinend etwas kleiner als bei der kaukasischen Form $M$. f. nehringi (S a t u n in, 1905). Sie nennen als vergleichbare Schädelmaße für $M$. $f$. intermedia: $\mathrm{Cbl}$ der $\hat{\sigma}(\mathrm{n}=5)$ $83,2 \mathrm{~mm}(81,2-84,6)$, der ㅇ $(\mathrm{n}=4) 77,9 \mathrm{~mm}(77,7$ bis 78,0$)$ und für die Jochbogenbreite der $\delta 51,4 \mathrm{~mm}(49,9$ bis 52,0$)$, der $q 47,0 \mathrm{~mm}(46,5$ bis $47,4)$.

Unsere Durchschnittswerte (Tab. 2) sind im Mittel kleiner als bei Heptner angegeben und liegen ganz deutlich unter den Angaben für M. f. nehringi. Heptner wiederholt mehrmals, daß die Subspeziessystematik wenig durchgearbeitet und revisionsbedürftig ist. Dies gilt besonders für die Subspeziesbezeichnungen leucolachnea (B l a n for d, 1879), altaica (S a t u n i n, 1914), ognevi ( $\mathrm{L}$ a p t e v, 1946), toufoeus ( $\mathrm{H}$ o g d s o $\mathrm{n}$, 1842) und kozlovi (O gnev, 1931), die auf ihren Synonymstatus bzw. ihre Verfügbarkeit noch einmal an größeren Serien überprüft werden und auch die Form intermedia einbeziehen müssen. Die weiße Kehlzeichnung variiert in gleicher Weise wie bei Materialien aus anderen geografischen Breiten (vgl. Abb. 3, Tafel IV).

An den 17 Schädeln aus der MVR konnte bei $2 \hat{o}$ und $3 \stackrel{q}{\text { das }}$ Fehle von eiem bzw. beiden $\mathrm{P}_{1}$ ermittelt werden, was durchaus vergleichbar mit der Häufigkeit dieser Zahnreduktion in europäischen Populationen ist.

Als bevorzugte Habitate haben wir in der MVR zerklüftete, nischenreiche Felshänge und Flußauen mit dichtem Auwaldgebüsch. Die ,undurchdringbaren" Weiden- und „Dornendickichte” am Bulgan-gol mit zahlerichen Verstecken im Bereich der Wurzelstöcke und aufgeschwemmtem Reisig sowie die Vielzahl verfallener und intakter Biberbaue 
Tabelle 1

Sammel- und Meßdaten von Martes foina aus der MVR.

\begin{tabular}{|c|c|c|c|c|c|c|c|c|c|c|c|c|c|}
\hline Nr. & Sex & Datum & & Herkunft & Gew & $\mathrm{Kr}$ & Schw & Hf & $\mathrm{Ohr}$ & $\mathrm{Cbl}$ & $\mathrm{Jb}$ & $\mathrm{oZr}$ & $\mathrm{uZr}$ \\
\hline 3236 & $0^{*}$ & 18.3.78 & Aimak & Gobi-Altai, Bugat, Bižin-gol & 1500 & 456 & 250 & 85 & 41 & 77,4 & 47,3 & 27,3 & 33,7 \\
\hline 3239 & $\sigma^{*}$ & 20.3 .78 & Aimak & Gobi-Altai, Bugat, Bižin-gol & 1600 & 430 & 240 & 76 & 40 & 82,8 & 50,0 & 29,5 & 35,0 \\
\hline 3241 & $0^{*}$ & 7.3.78 & Aimak & Gobi-Altai, Zogt, Gischuni-gol & 1250 & 420 & 250 & 79 & 39 & 84,1 & 51,0 & 29,5 & 35,6 \\
\hline 3242 & $\sigma^{*}$ & 2.3 .78 & Aimak & Gobi-Altai, Zogt, Choit-churemt & 1600 & 440 & 256 & 71 & 49 & 80,3 & 52,8 & 28,4 & 34,1 \\
\hline 3243 & $\sigma^{*}$ & 5.3 .78 & Aimak & Gobi-Altai, Zgot, Urd-churemt & 1500 & 431 & 230 & 73 & 42 & 80,2 & 49,7 & 27,8 & 34,5 \\
\hline 3245 & $\sigma^{*}$ & 25.2 .78 & Aimak & Gobi-Altai, Zogt, Gegeni-gol & - & 430 & - & - & - & 78,3 & 52,3 & 28,2 & 34,3 \\
\hline 3246 & $\sigma^{\pi}$ & 25.2 .78 & Aimak & Gobi-Altai, Zogt, Gegeni-gol & $-\overline{1}$ & 470 & - & - & - & 81,6 & 52,8 & 28,9 & 35,3 \\
\hline 24 & $0^{*}$ & 12.5 .74 & Aimak & Chovd, Bulgan, Bulgan-gol & 1180 & 445 & 255 & 83 & 42 & 79,5 & 53,2 & 27,5 & 34,3 \\
\hline 31 & $\sigma^{*}$ & 16.5.74 & Aimak & Chovd, Bulgan, Bulgan-gol & 1405 & 440 & 240 & 85 & 42 & 81,6 & 53 & 29,0 & 35,5 \\
\hline 156 & $\sigma^{*}$ & 9.6 .75 & Aimak & Chovd, Ulan-ul, $20 \mathrm{~km} \mathrm{NW}$ & 1330 & 440 & 270 & 88 & 42 & 82,0 & 52,9 & 28,8 & 35,2 \\
\hline 52 & $0^{*}$ & 14.5.78 & Aimak & Chovd, Bulgan, Bulgan-gol & 1445 & 430 & 250 & 89 & 44 & 81,3 & 53,0 & 28,3 & 34,1 \\
\hline 3237 & ? & 27.2 .78 & Aimak & Gobi-Altai, Zogt, Gegeni-gol & 112 & 430 & 230 & 70 & 39 & 79 & 46 & 26 & 32,9 \\
\hline 3238 & $q_{0}^{9}$ & 19.3 .78 & Aimak & Gobi-Altai, Bugat, Bižin-gol & & 400 & 205 & 78 & 39 & 76,0 & 47,2 & 27,2 & 33,2 \\
\hline 3240 & 운 & $\begin{array}{r}7.3 .78 \\
75.70\end{array}$ & Aimak & Gobi-Altai, Zogt, Gischuni-gol & 1000 & 415 & 240 & 72 & 34 & 78,8 & 50,4 & 27,8 & 34,7 \\
\hline 3244 & ?. & 25.2.78 & Aimak & Gobi-Altai, Zogt, Gegeni-gol & 1000 & 390 & 215 & 73 & 40 & - & - & 25,8 & 31,7 \\
\hline 173 & 웅 & 11.6 .75 & Aimak & Chovd, Ulan-ul, $20 \mathrm{~km} \mathrm{NW}$ Chovd & 950 & 400 & 240 & 81 & 40 & 76,9 & 46,6 & 27,4 & 32,9 \\
\hline 51 & 우 & 14.5 .78 & Aimak & Chovd, Bulgan, Bulgan-gol & 1150 & 420 & 240 & 80 & 41 & 77,1 & 50,1 & 26,8 & 32,3 \\
\hline
\end{tabular}


beherbergten im Mai der Jahre 1974, 1975 und 1978 nach den langen harten zentralasiatischen Wintern eine bemerkenswerte Anzahl von Steinmardern (Abb. 4, Tafel V). Über die exakte Populationsdichte kann jedoch wenig ausgesagt werden, da die gefangenen Tiere zufällig, in für andere Arten ,programmierte" Fallensysteme, gefangen wurden und keine speziellen Untersuchungen angestellt werden konnten. Regelmäßig waren Marderspuren neben denen von Wolf, Fuchs und Dachs an der Uferlinie des Flusses zu sehen.

Wir hatten niemals den Eindruck, daß der Steinmarder dort wo er vorkam, selten sei. Dies gilt auch auch für die Nachweise im Mongolischen Altai in den Somonen Zogt und Bugat sowie nördlich der Stadt Chovd im Ulan-ul-Massiv, das sich z.T. unmittelbar am Chovd-gol (Abb. 5, Tafel V) entlangzieht. Der jahrzehntelange Schutz dieser Art könnte sich

Tabelle 2

Mittelwerte der Körper- und Schädelmaße von Steinmardern aus der MVR.

\begin{tabular}{|c|c|c|c|c|c|c|c|c|}
\hline \multirow{2}{*}{$\mathrm{Maße}(\mathrm{g}+\mathrm{mm})$} & \multicolumn{4}{|c|}{$0^{x} 0^{x}$} & \multicolumn{4}{|c|}{ 우우 } \\
\hline & $\mathrm{n}$ & $\overline{\mathrm{x}}$ & $\min$ & $\max$ & $\mathrm{n}$ & $\overline{\mathrm{x}}$ & $\min$ & $\max$ \\
\hline Masse & 9 & 1423 & 1180 & 1600 & 6 & 1120 & 950 & 1500 \\
\hline Kopf-Rumpf-Länge & 11 & 439 & 420 & 470 & 6 & 409 & 390 & 430 \\
\hline Schwanz & 9 & 249 & 230 & 270 & 6 & 228 & 205 & 240 \\
\hline Hinterfuß & 9 & 81 & 71 & 89 & 6 & 76 & 72 & 81 \\
\hline $\mathrm{Ohr}$ & 9 & 42 & 39 & 49 & 6 & 39 & 34 & 41 \\
\hline Condylobasallänge & 11 & 80,8 & 77,4 & 84,1 & 5 & 77,6 & 76,0 & 79,1 \\
\hline Jochbogenbreite & 11 & 51,7 & 47,3 & 53,9 & 5 & 48,2 & 46,6 & 50,4 \\
\hline Zahnreihe oben & 11 & 28,5 & 27,3 & 29,5 & 6 & 26,9 & 25,8 & 27,8 \\
\hline Zahnreihe unten & 11 & 34,7 & 33,7 & 35,6 & 6 & 33,0 & 31,7 & 34,7 \\
\hline
\end{tabular}

durchaus auf die heutigen Bestände positiv ausgewirkt haben. Weitere Untersuchungen über die Populationsdichte sowie das gegenwärtige Areal müssen folgen, um gegebenenfalls den Steinmarder wieder in die Pelznutzung einbeziehen zu können.

Ohne über Datenmaterial zu verfügen nennt B a nnikov (1954) als Reproduktionsrate 2 bis 6 Junge, die je Weibchen im Februar/März geboren werden. Da Steinmarderweibchen nur über vier Milchzitzen verfügen, dürfte auch die Jungenzahl nur in seltenen Fällen darüber liegen. Die ersten Würfe fallen frühestens Ende März, aber hauptsächlich im April. Dies bestätigen zwei trächtige Weibchen aus dem mongolischen Material. Nr. 3240, gesammelt am 7.3.78, hatte jederseits einen Embryo von etwa $1 \mathrm{~cm}$ Länge. Das Weibchen Nr. 3238 hatte am 19.3.78 links einen Fötus und rechts 2 Embryonen inne. Diese Embryonen, alle drei Männchen, wogen jeweils 23 bis $24 \mathrm{~g}$, hatten eine Scheitel-Steißlänge von 90 bis $95 \mathrm{~mm}$ und eine Schwanzlänge von 35 bis $40 \mathrm{~mm}$. Die Geburtsmasse junger Steinmarder beträgt ca 
$30 \mathrm{~g}$ (H e p tne r \& N a u mov, 1974). Zur Lebensweise, besonders zur Nahrungsökologie, gab es aus der MVR bisher fast keine Angaben. Lediglich Bannikov (1954) untersuchte einige Exkremente von Martes foina aus dem Zagan-bogd-Gebirge im Transaltaigebiet und fand Reste von Cricetulus migratorius und Alticola roylei sowie Vogelfedern, Samen und andere Pflanzenreste.

Fast in allen Gebieten des Steinmarderareals kommen Kleinnagergesellschaften vor und brüten zahlreiche Kleinvogelarten, die besonders zur warmen Jahreszeit als Beute zur Verfügung stehen. Am Bulgan-gol kommt Dryomys nitedula in einer starken Population vor. Außerdem wurden Microtus oeconomus, Ellobius talpinus, Lagurus luteus, Apodemus spec., Mus musculus, sowie in den angrenzenden Halbwüsten und

\section{Tahelle 3}

Beutespektrum aus den Mägen und Darmtrakten der von Chotolchu untersuchten Steinmarder aus dem Mongolischen Altai.

\begin{tabular}{|c|c|c|c|c|c|c|c|c|c|}
\hline Beuteart & 3236 & 3237 & 3238 & 3239 & 3240 & 3241 & 3242 & 3243 & 3244 \\
\hline $\begin{array}{l}\text { Meriones spec. } \\
\text { Microtus spec. }\end{array}$ & + & & & & + & & & & \\
\hline $\begin{array}{l}\text { Alticola roylei } \\
\text { Alectoris chucar }\end{array}$ & + & + & + & & & & & + & \\
\hline Petronia petronia & & + & & & & & & & \\
\hline $\begin{array}{l}\text { Passeriformes } \\
\text { Tenebrionidae }\end{array}$ & + & & + & & & & & & \\
\hline $\begin{array}{l}\text { Tenebrionidae } \\
\text { Orthoptera }\end{array}$ & + & & & + & + & & & & \\
\hline Pflanzen & + & & + & + & & & & & \\
\hline $\begin{array}{l}\text { Schafwolle } \\
\text { Ziegenwolle }\end{array}$ & & & & + & & & & & + \\
\hline $\begin{array}{l}\text { Ziegenwolle } \\
\text { Kamelwolle }\end{array}$ & & & & & & & & & + \\
\hline $\begin{array}{l}\text { Stoffreste } \\
\text { unbestimmte }\end{array}$ & & & & & & & & & + \\
\hline Sägerreste & + & & & + & + & + & + & + & \\
\hline
\end{tabular}

Wüstengebieten Allactaga sibirica, Dipus sagitta, Meriones tamariscinus sowie einige Zwerghamsterarten und weitere Dipodiden nachgewiesen. Weitere Säugerarten sind Crocidura suaveolens, Erinaceus auritus, Lepus capensis tolai und Ochotona pallasii. Neuerdings ist auch die Bisamratte Ondatra zibethica am Bulgan-gol heimisch geworden (s. D a wa a, $\mathrm{St}$ ub b e und Dorzraa, 1977). Ob der Steinmarder auch Jungbibern gefährlich werden kann, ist bisher nicht belegt, bei den geringen Zuwachsraten von Castor fiber birulai and diesem Gewässer aber durchaus denkbar. Am Flußufer brüten Anser anser, Mergus merganser und mehrere Entenarten neben zahlreichen anderen Vogelarten. Auch dieDionenatter Elaphe dione kommt als Beutetier infrage. Aus der Vertebraten- und Entomofauna ist ein reiches Angebot vorhanden, das im 
Winter durch den Ausfall der Winterschläfer und Zugvögel sowie poikilothermen Formen naturgemäß stark reduziert ist. Aus den Mägen und Darmtrakten mehrerer Steinmarder des Mongolischen Altai konnte Chotolchu nachfolgendes Beutespektrum bestimmen (Tab. 3). Als maximale Masse konten $60 \mathrm{~g}$ als Mageninhalt ermittelt werden. Diese Angaben werden ergänzt durch die Aufsammlung und Auswertung von 194 Exkrementen von Steinmardern aus den Somonen Zogt, Bugat und Altai (Tab. 4). Danach haben Pfeifhasen, Bergwühlmäuse der Gattung

Tabelle 4

Analyse von 194 Steinmarderexkrementen aus dem Altai durch Chotolchu und qualitative Häufigkeit der einzelnen Nahrungselemente

\begin{tabular}{|c|c|c|c|c|c|}
\hline Art & $\mathrm{n}$ & $\%$ & Art & $\mathrm{n}$ & $\%$ \\
\hline Ochotona pallasii & 50 & 25,7 & Eidechsen & 1 & 0,5 \\
\hline Allactaga spec. & 1 & 0,5 & Carabidae & 5 & 2,5 \\
\hline Mus musculus & 2 & 1,0 & Tenebrionidae & 2 & 1,0 \\
\hline Phodopus spec. & 1 & 0,5 & Chrys melidae & 1 & 0,5 \\
\hline Cricetulus spec. & 5 & 2,5 & Buprestidae & 1 & 0,5 \\
\hline Meriones spec. & 10 & 5,1 & Käferlarven & 2 & 1,0 \\
\hline Alticola roylei & 108 & 55,6 & Orthopteren & 28 & 14,4 \\
\hline Microtus oeconomus & 7 & 3,5 & Schafwolle & 9 & 4,6 \\
\hline Microtus spec. & 17 & 8,7 & Rinderhaare & 1 & 0,5 \\
\hline Passeriformes & 33 & 17,0 & Pflanzensamen & 2 & 1,0 \\
\hline Alectoris chucar & 1 & 0,5 & Pflanzen & 15 & 7,7 \\
\hline Vogeleier & 3 & 1,5 & unbestimmte Säugerreste & 8 & 4,1 \\
\hline
\end{tabular}

Alticola und Passeriformes die größte Bedeutung in der Ernährung. Der Nachweis von Haustierwolle in den Mägen und Exkrementen läßt die Aufnahme von Aas bzw. Schlachtabfällen vermuten.

\section{LITERATUR}

1. B a keev N. N., 1972: On the relation of the distribution of martens (subgenus Martes Pinel) in the USSR to binc'imatic foctors. Bull. Mosc. Soc. Nat., Biol. Ser., 77, 2: 5-15. [in Russian with English summ.].

2. B a nnikov A. G., 1954: Mlekopitajuščie Mongolskoi Narodnoi Respubliki. Moskva.

3. Dawa a N., Stubbe M. \& Dorzraa O., 1977: Die Bisamratte Ondatra zibethica (L., 1758) in der Mongolischen Volksrepublik. Beitr. Jagd- und Wildforschung, 10: $342-352$.

4. Dula mseren S., 1970: Mongol orny chochton amtan todorchoiloch butschig. Ulan-Bator.

5. Dulamseren S. \& Chotolchu N., 1972: Promyslovyje mlekopitajuščie. [In: Dulma A. \& Schagdarsuren O. "Promyslovyje životnyje MNR i ich ochrana«]. Ulan-Bator: 146-214. 
6. Heptner V. G. \& Na umov N. P., 1974: Die Säugetiere der Sowjetunion. Jena. Bd. II.

7. Ryabov L. S., 1976: The stone marten (Martes foina) and the pine marten (Martes martes) in the Voronezh oblast. Bull. Mosc. Soc. Nat., Biol. Ser., 81, 4: 24 -37. [ In Russian with English summ.].

8. Strog a n ov S. U., 1962: Zveri Sibiri. Chiščnye. Moskva.

Accepted, August 20, 1979.

Namshil CHOTOLCHU, Michael STUBBE i Naniragijn DAWAA

\section{KUNA KAMIONKA W MONGOLII}

\section{Streszczenie}

Martes foina (Erxle be n, 1777) jest wielką rzadkością w kolekcjach ssaków z Mongolii. Oprócz biometrycznych danych z 17 osobników, zebranych między 1974 i 1978, uzyskano nowe informacje o rozprzestrzenieniu i ekologii $M$. foina w Mongolii. Rozmieszczenie kuny kamionki obejmuje obszar od południowych partii Mongolii do północnej strefy $\mathrm{z}$ izotermą stycznia około $-25^{\circ} \mathrm{C}$. Występuje regularnie i pospolicie w poludniowo-zachodniej Mongolii w Bulgan-gol, w Ałtaju mongolskim w regionach Zogt i Bugat, oraz około $20 \mathrm{~km}$ na północ od miasta Kobdo w górach Ulan-ul (Tabele 1, 2; Ryc. 1-5). Pokarm jej stanowią różne małe ssaki i ptaki (Tabele 3,4). Rozród zaczyna się na przełomie lutego i marca. 


\section{TAFELBESCHREIBUNG}

Tafel IV

Abb. 3. Kehlzeichnung mongolischer Steinmarder, oben vom Bulgan-gol, unten aus dem Mongolischen Altai.

Tafel V

Abb. 4. Biotop von Martes foina am Bulgan-gol in der SW-Mongolei. Abb. 5. Das Ulan-ul-Gebirge am Chovd-gol, $20 \mathrm{~km} \mathrm{NW}$ der Stadt Chovd. 


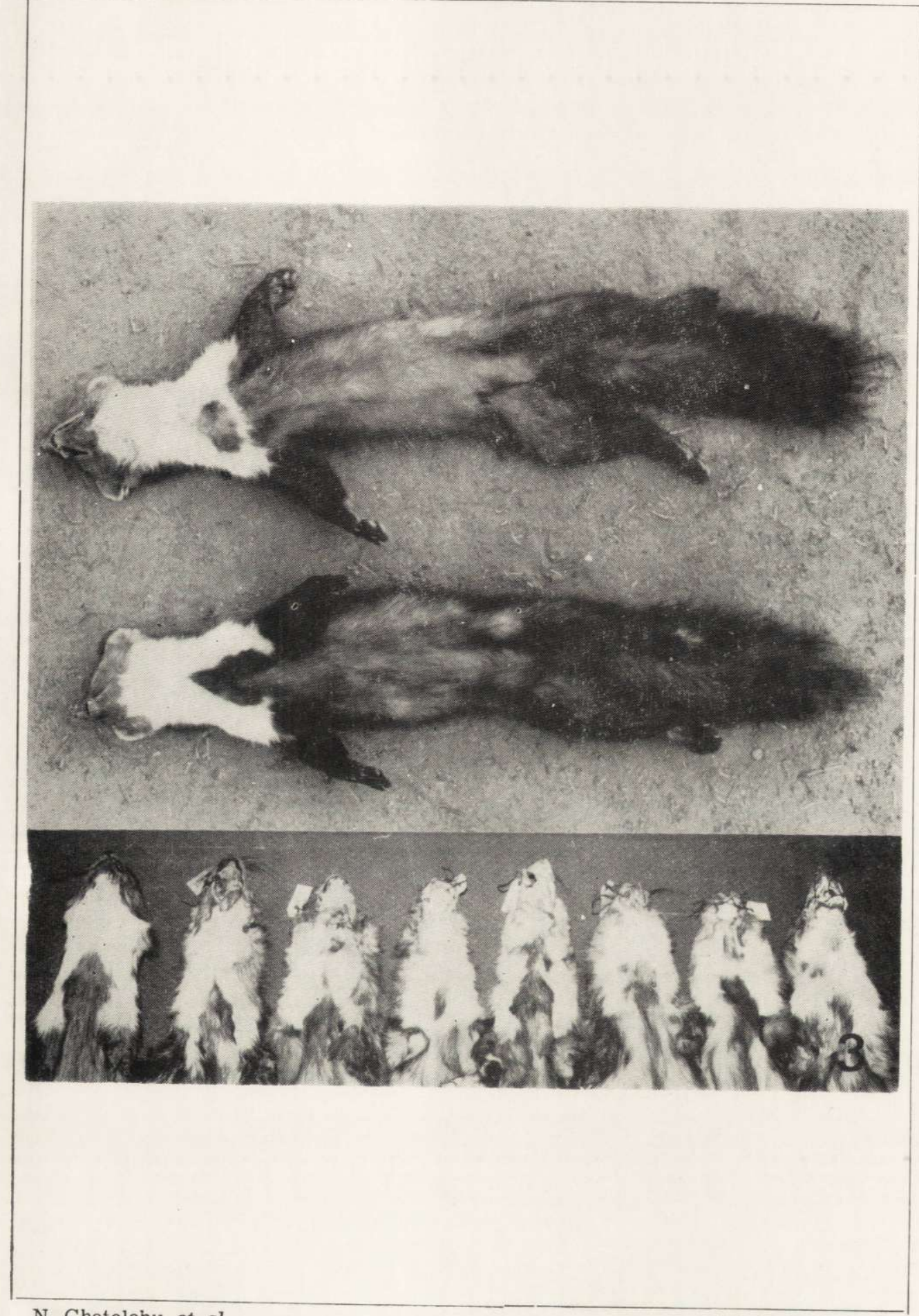

N. Chotolchu et al. M. Stubbe phot. 


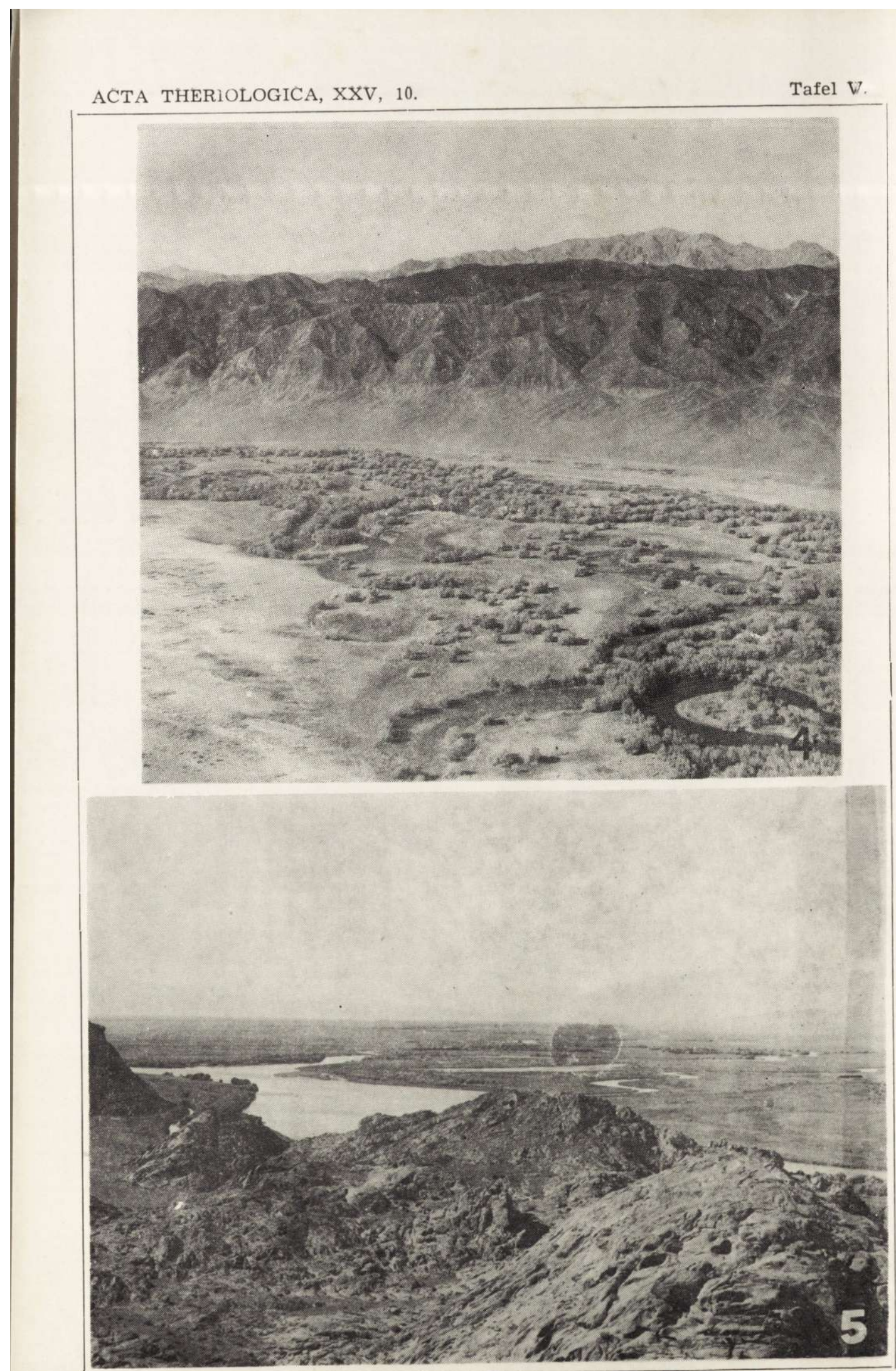

M. Stubbe phot. 